\title{
An Application of Molecular Genotyping in Mice
}

\author{
Lara A. Underkoffler ${ }^{1 *}$, Joelle N. Collins ${ }^{1}$, Jonathan D. Choi ${ }^{2}$ and Rebecca J. Oakey ${ }^{2}$ \\ ${ }^{1}$ Division of Human Genetics, The Children's Hospital of Philadelphia, Philadelphia, PA 19104, USA. \\ ${ }^{2}$ Division of Medical Genetics, GKT School of Medicine, $8^{\text {th }}$ Floor Guy's Tower, London, SE1 9RT, UK. \\ *To whom correspondence should be addressed: Lara Underkoffler, underkoff@email.chop.edu
}

Submitted: March 19, 2003; Revised: March 27, 2003; Accepted: April 4, 2003; Published: May 1, 2003

Indexing terms: Genotyping, Nondisjunction, Simple Sequence Repeat Polymorphism.

\begin{abstract}
Microsatellite markers are simple sequence repeats within the mammalian genome that can be used for identifying disease loci, mapping genes of interest as well as studying segregation patterns related to meiotic nondisjunction. Different strains of mice have variable CA repeat lengths and PCR based methods can be used to identify them, thus allowing for specific genotypes to be assigned. Molecular genotyping offers such identification at any developmental stage, which allows for a broad range of anomalies to be studied. We studied chromosomal segregation in relation to nondisjunction in earlygestation mouse embryos using molecular genotyping. Information on the parental origin as well as the number of chromosomes a given progeny carried was obtained in our analysis.
\end{abstract}

\section{INTRODUCTION}

\section{Molecular markers}

Microsatellite markers are generated from simple sequence repeats (SSRs) within the eukaryotic genome and are utilized via molecular genotyping for scoring genetic information (1). This simple PCR-based method is useful for mapping genes of interest, identifying disease loci, and in this case, studying individual chromosome segregation patterns in mice and relating them to meiotic nondisjunction. A SSR is a genomic element consisting of one to several nucleotides repeated in tandem. The most common and identifiable mouse SSR consist of a series of CA repeats (2). It is estimated that the mouse genome has approximately 100,000 CA repeat blocks, yielding an average of 1 locus per $30 \mathrm{~kb}(3)$. These repeats are generated during recombination or replication as slippage, and mispairing can often occur within these regions (2). Variability in CA repeat length frequently occurs between inbred strains of mice and only occasionally do differences exist within a strain. PCR-based methods can be used to distinguish these polymorphic markers using primers located in unique flanking sequence, thus allowing strain specific genotypes to be scored (See Figure 1). It should be noted that genotyping outbred mice or mice of mixed backgrounds is possible, but may require the use of a more extensive marker panel.

Schematic of Simple Sequence Repeat PCR

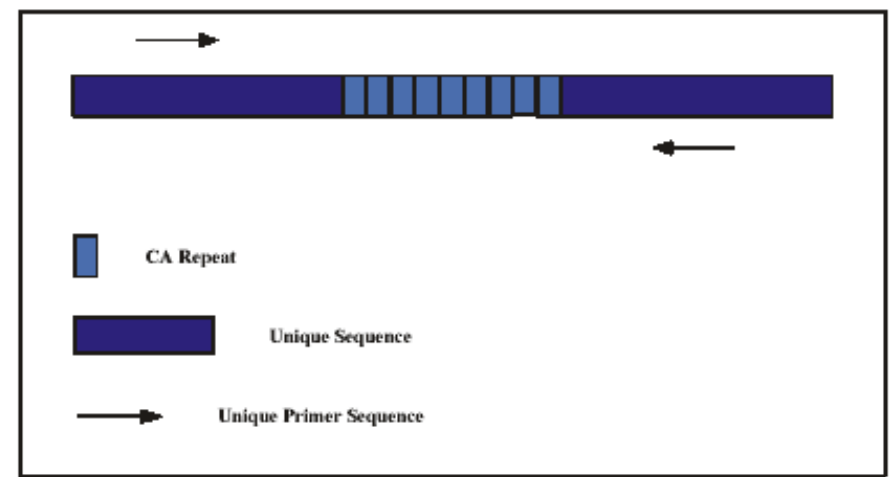

Fig. 1: A schematic representation of a CA repeat block flanked by unique DNA sequences in the mouse genome. CA repeats occur in blocks that are usually the same length within a strain of inbred mice. However, these blocks can vary in length between strains of mice and this size polymorphism can be detected via PCR from primers designed to amplify across the block from unique sequence. These types of markers are termed simple sequence repeat markers (SSRs). These markers can be used to identify the parental origins of chromosomes in progeny from an inter-strain or inter-sub specific cross.

\section{Phenotypic markers}

Before molecular markers were in common use, visible markers were used to identify chromosomal segregation patterns associated with chromosome anomalies and phenotypic abnormalities (4-6). Mice and embryos with uniparental inheritance of specific chromosomes were identified using genetic markers (7). Visible markers, while having been indispensable for the wealth of information 
generated for many decades on chromosome segregation, have two major drawbacks. One is that eye color cannot be detected until mid-gestation (around $11.5 \mathrm{dpc}$ ) and other visible phenotypes often are not in evidence until after birth. For studies of early developmental phenotypes or lethalities, it is essential to be able to identify the chromosome involved in the aneupoloidy or uniparental inheritance at any selected time during development. The second is that a phenotypic marker limits studies to regions of the genome that harbor genes with visible phenotypes. The use of molecular genotyping to determine the parental origin and number of chromosomes in a given embryo vastly expands the power of chromosome segregation analysis across the genome. Firstly, there is no limit to the age of embryos for molecular genotyping and secondly, markers are available throughout the mouse genome for genotyping between different strains of mice. Finally, genotyping by PCR is more efficient than chromosomal karyotyping or phenotype examination allowing more rapid sample processing (8).

\section{Nondisjunction}

We have used extensive molecular genotyping in mice to study non-disjunction events in mice with a Robertsonian chromosome. The parent of origin of a given chromosome or region may have an effect on the development or viability of the offspring. Studies analyzing the developmental consequences of uniparental inheritance of mouse chromosomes have defined imprinted regions throughout the mouse genome $(6,9)$ largely using Robertsonian translocation chromosomes. The inheritance of both copies of a chromosome from a single parent is termed uniparental disomy (UpDi). Identification of UpDi is facilitated by the use of molecular tools to determine the parental identity of individual mouse chromosomes or chromosomal regions. This is particularly useful where large numbers of mice are studied in strains that lack convenient phenotypic markers. The population of mice in our study carried a Robertsonian translocation, this is a metacentric chromosome which results from the fusion of two normally acrocentric mouse chromosomes. Robertsonian translocations exist in wild populations of mice mostly in Western Europe and North Africa (10-12) and have been transferred onto inbred backgrounds in the laboratory (http://www.jax.org/index.html). Mice that are homozygous for a given Robertsonian translocation give rise to balanced gametes, but mice heterozygous for a Robersonian translocation can give rise to both balanced and unbalanced gametes (5). In rare instances, the fusion of two complementary unbalanced gametes gives rise to a chromosomally balanced viable zygote with 2 maternal or 2 paternal copies of the chromosome arms involved in the translocation (13) and Figure 2. Complementation studies like these in mice have been used extensively to study nondisjunction of chromosomes (4). The frequency of progeny with UpDi or chromosomal imbalance will be low, depending on the chromosome anomaly in question and it is essential that a marker system be available to distinguish the above progeny from those with normal chromosome complements. Historically, crosses have relied on phenotypic genetic markers to identify non-complementation of selected chromosomes and regions, molecular markers however, provide a more versatile way of identifying parental origin.

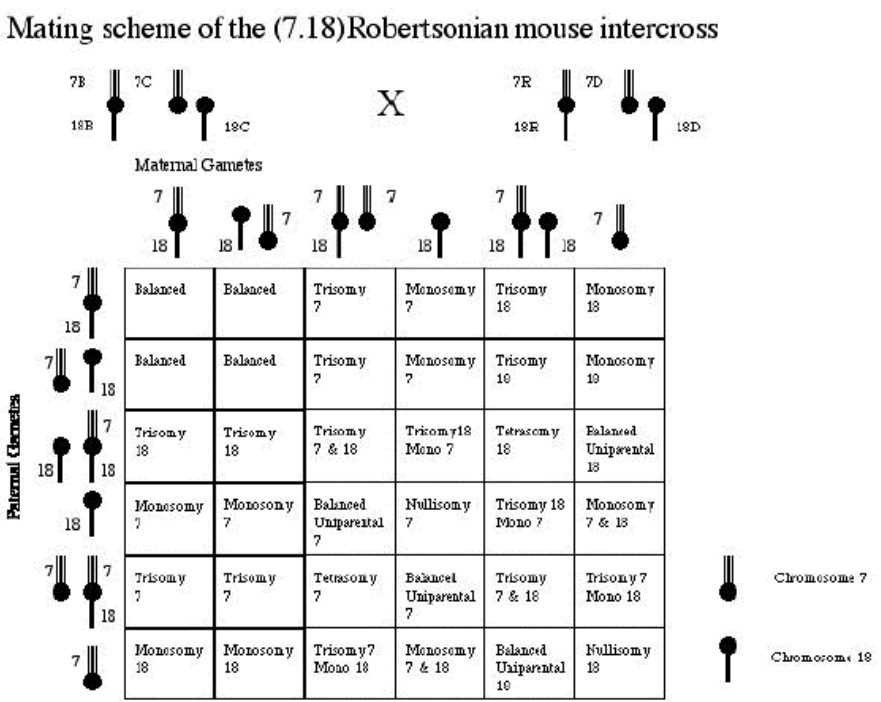

Fig. 2: A schematic diagram representing the potential progeny resulting from a cross between strains of mice heterozygous for the Robertsonian (7.18) chromosome. The categories that survive to the time of embryo harvest are normal chromosome complements, balanced chromosome complements, trisomies, double trisomies and uniparental disomies for both chromosomes 7 \& 18. Monosomies and tetrasomies were not detected. The maternal and paternal gametes are indicated in one direction but the crosses are performed in both directions and so embryos are derived from both maternal and paternal combinations of gametes for each strain.

We intercrossed mice heterozygous for a (7.18) Robertsonian translocation (14) and have genotyped 1,812 embryos from 364 litters with known parental origin, strain and age (15). The key feature of the strains of mice used in these types of studies is that they are inbred strains with defined genetic markers. As a result, DNA polymorphisms between strains of mice are readily identified and the number and parent of origin of each chromosome are readily determined. Thus aneuploid progeny from crosses involving trisomy or monosomy as well as uniparental inheritance for a particular chromosome can be easily identified (Figures 2 and 3 ) and the frequency of nondisjunction determined.

\section{MATERIALS AND METHODS}

\section{Mouse intercross scheme}

We produced two strains of mice heterozygous for the Robertsonian chromosome $\mathrm{Rb}(7.18)$ by mating homozygous $\mathrm{C} 57 \mathrm{BL} / 6 \mathrm{Jei}-\mathrm{Rb}(7.18) 9 \mathrm{Lub}$ (denoted as BXB)with $\mathrm{C} 3 \mathrm{H} / \mathrm{HeJ}$ or Mus musculus Castaneous (denoted as CXC) and $\mathrm{Rb}(2.8)$ 2Lub / (7.18) 9Lub (denoted as RXR) with DBA/2J (denoted as DXD) to yield BXC or CXB and RXD or DXR heterozygotes, respectively. The Robertsonian mice were 
obtained from The Robertsonian Resource at The Jackson Laboratory, Bar Harbor, Maine. As explained previously, the gametes of these Robertsonian heterozygotes can be balanced or unbalanced. When the $\mathrm{Rb}$ heterozygotes are intercrossed they can produce zygotes that have duplications ( 2 copies) and deficiencies ( 0 copies) of chromosomes 7 or 18 . We have detected offspring with normal, trisomic and UpDi genotypes using polymorphic markers for each individual mouse strain used in the cross (Figure 3).

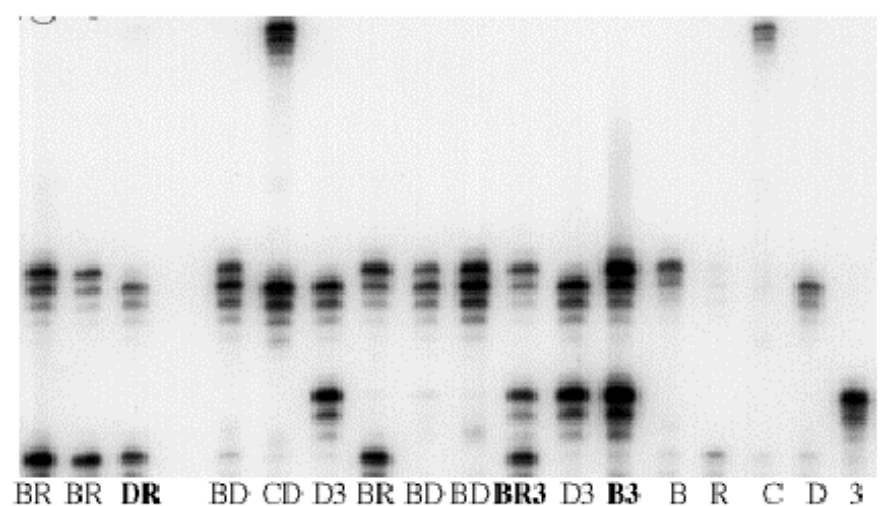

Fig. 3: A denaturing polyacrylamide genotyping gel separating radioactive PCR products from various mouse strains. The polymorphic parental strains of mice are shown in lanes marked B (C57BL/6JEi-Rb(7.18)9Lub), R $(\mathrm{Rb}(2.8) 2 \mathrm{Lub} /$ (7.18)9Lub ), C (M.m. Castaneus), D (DBA/2J) and 3 $(\mathrm{C} 3 \mathrm{H} / \mathrm{HeJ})$. Genotypes in bold type-face indicate aneuploid genotypes. BR3 is a trisomy (it shows three chromosome 7 bands); DR \& B3 are both uniparental disomies (UpDi) since the mother of DR was a DXR animal and the mother of B3 was a BX3 animal. No paternal chromosome 7 was present in the genotypes of these two animals. All the other genotypes indicate inheritance of chromosome 7 from both parents and confirm a normal chromosomal inheritance for these embryos.

Note that in practice, not all of the progeny are viable and thus will not be represented in the outcome. Monosomies (only one copy of a chromosome pair) and tetrasomies (4 copies) are non-viable at the embryonic ages scored. The categories that survive to the time of embryo harvest are normal chromosome complements, balanced chromosome complements, trisomies ( 3 copies of the same chromosome) including double trisomies and uniparental disomies for both chromosomes $7 \& 18$. The age of harvest was determined empirically by harvesting embryos at $10.5 \mathrm{dpc}$ and working backwards to $8.5 \mathrm{dpc}$ to determine the latest stage of development that embryos with the desired genotypes (trisomies of chromosomes 7 and 18 and $\mathrm{UpDi}(7)$ or $\mathrm{UpDi}(18)$ ) were present and phenotypically normal. This was determined by inspection of the embryos at the day of harvest and by counting the number of somites. The embryos were genotyped for the $\mathrm{Rb} 2.8$ chromosome present only in the RXR strain and not the BXB strain (unplublished data) and its segregation had no measurable effect on the phenotype of the trisomy 7 or 18 embryos scored at $8.5 \mathrm{dpc}$ (14). Only one embryo with trisomy 18 also had trisomy 8 and this embyro was normal phenotypically. No trisomy 18 embryos had trisomy 2 also, which is not surprising since a double trisomy would be a rare event.

\section{DNA preparation}

A sample of tissue from the tail tip of each embryo was procured from $8.5 \mathrm{dpc}$ mouse embryos. DNA was isolated using $100 \mu \mathrm{l}$ of Lysis buffer containing $100 \mathrm{mM} \mathrm{KCl}, 20 \mathrm{mM}$ Tris(pH8.3), $5 \mathrm{mM} \mathrm{MgCl}_{2}, 1.4 \mu \mathrm{M}$ sodium dodecyl sulfate, $40 \mathrm{mM}$ dithiothreitol, $2 \mathrm{mg} / \mathrm{ml}$ gelatin and $0.1 \mathrm{mg} / \mathrm{ml}$ proteinase $\mathrm{K}$ solution. The samples were vortexed, and centrifuged briefly to bring contents to the bottom of each tube, then incubated at $37^{\circ} \mathrm{C}$ for 1 hour. Each tube was immersed in boiling water for 5 minutes for proteinase $\mathrm{K}$ inactivation. This is extremely important as proteinase $\mathrm{K}$, if present in the PCR reaction, will digest the DNA polymerase. Samples were stored at $-20^{\circ} \mathrm{C}$ until needed.

\section{Primers and PCR}

In this study, we looked at markers on chromosomes 7 and 18. Research Genetics offers mouse MapPairs ${ }^{\circledR}$ Microsatellite Markers to detect SSRs throughout the entire genome. Markers and PCR product sizes are available for several strains of mice on their website (ftp://ftp.resgen.com/pub/mappairs) (Table 1).

Table 1: A table listing Simple Sequence Repeat sizes of specific Mit Markers ${ }^{\mathrm{TM}}$ across different strains of mice

\section{Listing of SSR size versus mouse strain}

Locus Mouse strain and SSR size in bp

\begin{tabular}{lccccc}
\hline & B6 & Cast & C3H & DBA & Rb \\
D7Mit25 & 110 & 144 & 96 & 108 & 88 \\
D7Mit222 & 147 & 178 & 123 & 123 & 155 \\
D18Mit14 & 105 & 125 & 107 & 96 & 112 \\
D18Mit87 & 144 & 176 & 140 & 152 & 130 \\
\hline
\end{tabular}

The primary chromosome 7 marker used was D7Mit25 with D7Mit222 as the secondary marker being used for confirming aneuploid genotypes. Primarily D18Mit14 was used for chromosome 18 genotyping with D18Mit87 as the marker of choice for confirmation of genotype. The use of a second marker is helpful, especially with ambiguous first scores and for confirming aneuploidy. Using PCR with radioactively endlabeled primers (1), $1 \mu 1$ samples of DNA were genotyped. The $10 \mu$ reaction mix contained 0.5 units of AmpliTaq DNA polymerase (Perkin Elmer) with manufacturer's buffer in the presence of $0.4 \mathrm{mM}$ dNTPs and both unlabeled primers at a concentration of $0.22 \mu \mathrm{M}$, and one end-labeled primer at an approximate concentration of $0.06 \mu \mathrm{M}$. Primers were endlabeled with $\gamma{ }^{32} \mathrm{P}$ ATP using polynucleotide kinase (16). Amplification conditions were: $3 \mathrm{~min}$ at $94^{\circ} \mathrm{C}$ followed by 30 cycles of $94^{\circ} \mathrm{C}$ for $15 \mathrm{sec}, 55^{\circ} \mathrm{C}$ for $30 \mathrm{sec}$, then $72^{\circ} \mathrm{C}$ for 30 sec, followed by a final $72^{\circ} \mathrm{C}$ extension for $7 \mathrm{~min}$. To each completed reaction tube $2 \mu$ of formamide loading buffer containing bromophenol blue and xylene cyanol was added. 
The samples were then heated at $95^{\circ} \mathrm{C}$ for $5 \mathrm{~min}$ and $3 \mathrm{ul}$ of each sample were loaded onto an $8 \%$ denaturing polyacrylamide gel. Microsatellite markers often differ in size by only 1 to 10 base pairs and thus need to be separated on polyacrylamide gels. The samples were electrophoresed on a sequencing style electrophoresis apparatus until the PCR products were clearly separated, frequently around two hours at $10 \mathrm{~V} / \mathrm{cm}$ for the SSRs we were assaying, but dependant on the expected PCR product sizes. Gels were dried for one hour on a vacuum gel dryer, exposed to Kodak X-omat film overnight and the genotypes scored by two different individuals. Any samples with discordant scores were repeated with the secondary primer set as mentioned above as were all aneuploid individuals.

\section{RESULTS AND DISCUSSION}

\section{Tissue sampling}

The key features of the experimental design require careful sample collection, good DNA preparation, identification of genetic markers that are polymorphic by a simple PCR reaction and an appropriate detection system. Sample collection was performed consistently to avoid contamination of embryonic samples with maternal membranes, as this would have jeopardized correct determination of chromosomal inheritance. This problem is exacerbated because of the small size of mouse embryos at $8.5 \mathrm{dpc}$. Care was also taken to avoid cross contamination between embryos within a litter by washing forceps in fresh phosphate buffered saline in between each dissection. Flash tissue freezing was practiced in order to prevent nucleic acid degradation. High quality DNA was obtained using classic lysis methods (17). As stated above, if DNA is prepared with a proteinase $\mathrm{K}$ based lysis method, care must be taken to inactivate the proteinase $\mathrm{K}$ prior to $\mathrm{PCR}$ amplification. An alternative method of DNA preparation is now available with commercial DNA isolation kits. We have recently had success with Qiagen's DNAeasy kit ${ }^{\circ}$, however a variety of manufacturers offer similar DNA preparation kits.

\section{Polymorphic markers and gel electrophoresis}

Defined polymorphic SSR markers have provided the means to assay strain information using PCR (Figure 3). SSR polymorphism size versus strain information is readily obtained from the Research Genetics ${ }^{\circledR}$ online database (http://www.resgen.com). The PCR reaction itself is simple and straightforward, requiring standard PCR reagents and a radioactively end-labeled oligonucleotide primer. There are a variety of different detection methods available to view genotyping results. First, consider the size of the PCR products. The use of radioactive isotope coupled with electrophoresing on a polyacrylamide gel is preferable with product size differences of 1 to 10 base pairs, as is often the case with SSRs in the mouse genome. The resolution is higher with the use of polyacrylamide gels compared to agarose gels and thus small differences in size can be easily scored. Genotyping may, however, be performed without isotope if the PCR product sizes can be distinguished with the use of agarose gel electrophoresis. We chose to work with denaturing polyacrylamide gels because the results were cleanest and most discernable, while nondenaturing polyacrylamide gels were found to be frequently equivocal in our hands. It should be noted that either denaturing or non-denaturing poylacrylamide gels could be used to discern PCR products differing by $10 \mathrm{bp}$ or less. Although we obtained the most reproducible results using denaturing polyacrylamide gels, we occasionally experienced difficulties. Gel resolution varied from time to time due to the instability of reagents such as ammonium persulfate and acrylamide. Another potential problem for scoring band sizes is the slight leakage of samples from one lane into adjacent lanes, leading to ambiguous or inaccurate genotypes especially in scoring trisomic individuals, so care must be taken when removing the gel comb so as to not affect the wells. It is also important for multiple independent reviewers to score the gels for consistency. Ambiguous genotypes were repeated using a different marker set. Multiplexing is an option when reading more than one marker at the same time per sample per lane and when the product sizes are different. PCR products are sometimes biased in multiplexed reactions due to problems with primer and template stoichiometry, so combining different PCR products prior to gel loading may alleviate this issue. Alternatively, a second loading of the same primer pair at a staggered interval can be used.

We have found that overall the process works very well, keeping these suggestions and guidelines in mind, large sets of data can be generated in a short period of time. Due to the generation of large amounts of genotyping, it becomes necessary to use proper statistical analysis. Consequently, organization of data in a logical manner should be considered prior to the study being performed. We found it necessary to record not only the embryonic genotypes, but also the maternal and paternal strains and ages. Careful statistical analysis allows for the making of sound conclusions based on the data generated. It is also imperative to project data collection needs at the beginning of any large-scale experiment or genotyping study.

\section{Statistical analysis of genotyping data}

We have performed genotyping of specific mouse crosses on a defined genetic background allowing us to identify particular parental alleles in offspring and thus infer chromosomal segregation in relation to meiotic nondisjunction. In our case, we wished to analyze the frequency of nondisjunction as a function of various properties of the parental animals. The parameters studied include maternal and paternal strain background, age of parents and chromosomal parent of origin. It is therefore necessary to record not only the embryonic genotypes, but also the measurement of the various physiological properties of the parental animals to be used in 
the statistical correlation analysis for nondisjunction. When looking for effects using litters of mice, it is important to consider that as the sampling of embryos naturally groups the data as litters, one might expect effects to be observed not only across an entire population, but perhaps that certain effects are also clustered within litters. This can be detected using a likelihood-ratio test comparing two regression models, one with and one without, a random effects parameter to account for litters (15). If evidence of clustering is detected, all further correlations must be identified accounting for the clustering nature of the observations. Generalized Estimator Equations (GEE) provide a practical method for the analysis of such correlated data $(15,18)$.

For genetic data assuming Mendelian inheritance (no gamete or post-fertilization selection) the expected ratios can be easily determined using Chi-square statistics and allowing associated $\mathrm{P}$ values to be calculated. A 95\% confidence interval is typically chosen and statistical significance of correlation determined accordingly. Prior to beginning the experiment, the minimal sample size necessary to reject the null hypothesis can be calculated at the desired confidence interval, as well as the number of expected cases of each genotype, assuming Mendelian inheritance. Understanding the sample size required to prove or disprove the hypothesis is important before undertaking a genotyping project, and consultation with a biostatistician or an epidemiologist is recommended.

In summary, due to the ease and robustness of the technique, the advent of molecular genotyping has vastly increased the utility and size of data sets that can be generated compared to karyotyping or phenotyping. This expands the opportunities for studying heritable conditions such as nondisjunction frequencies (15) and imprinting (as a consequence of uniparental inheritance of chromosomes or regions) on mouse development and viability, and places such studies on a solid statistical foundation.

\section{REFERENCES}

1. Dietrich W. A genetic map of the mouse suitable for typing intraspecific crosses. Genetics 1992; 131:423-447.

2. Silver L. Mouse Genetics. New York: Oxford University Press, Inc., 1995.

3. Hamada H, Petrino MG, Kakunaga T. A novel repeated element with Z-DNA-forming potential is widely found in evolutionarily diverse eukaryotic genomes. Proceedings of the National Academy of Sciences 1982b; 79:6465-6469.

4. Searle AG, Beechey CV. The use of Robertsonian translocations in the mouse for studies on non-disjunction. Cytogenet Cell Genet 1982; 33:81-87.

5. Searle AG, Beechey CV. Complementation studies with mouse translocations. Cytogenet Cell Genet 1978; 20:282303.

6. Searle AG, Beechey CV. Noncomplementation phenomena and their bearing on nondisjunction events in
Aneuploidy, V.L. Dellarco, P.E. Voytek, and A. Hollaender, eds. New York: Plenum Press, 363-376, 1985.

7. Cattanach BM, Beechey C. Genomic imprinting in the mouse: possible final analysis in Genomic imprinting: frontiers in molecular biology, W. Reik and A. Surani, eds. Oxford: Oxford University Press, 1997.

8. Williamson CM et al. Glomerular-specific imprinting of the mouse Gsalpha gene: How does this relate to hormone resistance in albright hereditary osteodystrophy? Genomics 1996; 36(2):280-287.

9. Cattanach BM. Parental origin effects in mice. $J$ Embryol Exp Morph 1986; 97:137-150.

10. Redi CA, Capanna E. Robertsonian heterozygotes in the house mouse and the fate of their germ cells in The cytogenetics of mammalian autosomal rearrangements, A. Daniel, ed. New York: Wiley-Liss, 315-359, 1988.

11. Capanna $\mathrm{E}$ et al. Robertsonian metacentrics in the mouse. Chromosoma 1976; 58:341-353.

12. Gropp A, Winking H. Robertsonian translocations: cytology, meiosis, segregation pattern and biological consequences of heterozygosity in Biology of the House Mouse, R.J. Berry, ed. Academic Press: New York and London. 141-181, 1981.

13. Searle AG, Ford CE, Beechey CV. Meiotic disjunction in mouse translocations and the determination of centromere position. Genetical Research 1971; 18:215-235.

14. Oakey RJ et al. Nondisjunction rates and abnormal embryonic development in a mouse cross between heterozygotes carrying a $(7,18)$ Robertsonian translocation chromosome. Genetics 1995; 141:667-674.

15. Underkoffler LAM, Mitchell LE, Localio AR, Marchegiani SM, Morabito J, Collins JN, Oakey RJ. Molecular analysis of nondisjunction in mice heterozygous for a robertsonian translocation. Genetics 2002; 161:1219-1224.

16. Tabor S. Enzymatic manipulation of DNA and RNA in Current Protocols in Molecular Biology, F.M. Ausubel et al., eds. 1994, Greene Publishing Associates Inc. and Wiley \& Sons Inc., 3.10.2-3.10.4, 1994.

17. Maniatis T. Recombinant DNA in Cell Biology, D.M. Prescott, ed. New York: Academic Press: 1980.

18. Liang P, Pardee AB. Differential display of eukaryotic messenger RNA by means of the polymerase chain reaction. Science 1992; 257:967-971. 


\section{PROTOCOLS}

\section{Equipment}

- Rainin Pipets (P10, P20, P200, P1000)

- Eppendorf Microcentrifuge

- Sorval RT6000B Centrifuge

- $95^{\circ} \mathrm{C}$ Heat Block

- MJ Research PTC-200 Thermal Cycler

- VWR-600 Power Supply

- Life Technologies Model2 Sequence Apparatus

- Gibco/BRL Sharkstooth $0.4 \mathrm{~mm}$ comb.

\section{Reagents and Methods}

\section{DNA Preparation}

\begin{tabular}{lll}
\hline 2X PCR Lysis Buffer & Volume & Final concentration \\
\hline Water & $7.23 \mathrm{ml}$ & $100 \mathrm{mM}$ \\
$1 \mathrm{M} \mathrm{KCl}$ & $1 \mathrm{ml}$ & $20 \mathrm{mM}$ \\
$1 \mathrm{M} \mathrm{Tris}(\mathrm{pH} 8.3)$ & $200 \mu \mathrm{l}$ & $5 \mathrm{mM}$ \\
$1 \mathrm{M} \mathrm{MgCl} 2$ & $50 \mu 1$ & $1.4 \mu \mathrm{M}$ \\
$1 / 100020 \%$ SDS & $20 \mu 1$ & $40 \mathrm{mM}$ \\
$1 \mathrm{M} \mathrm{DTT}$ & $400 \mu \mathrm{l}$ & $40 \mathrm{mM}$ \\
$2 \%$ Gelatin & $1 \mathrm{ml}$ & $2 \mathrm{mg} / \mathrm{ml}$ \\
\hline
\end{tabular}

1. Mix the buffer thoroughly and aliquot $495 \mu 1$ per eppendorf tube.

2. Store at $-20^{\circ} \mathrm{C}$.

3. When needed add $5 \mu 1$ of proteinase $\mathrm{K}(10 \mathrm{mg} / \mathrm{ml})+500 \mu 1$ water to tube of $2 \mathrm{X}$ Buffer and mix well.

4. For each DNA sample, add $100 \mu \mathrm{l}$ of mixture and vortex well, spin and incubate at $37^{\circ} \mathrm{C}$ for 1 hour. Boil samples at $100^{\circ} \mathrm{C}$ for 5 minutes.

SSR Analysis

\section{Primer End-labeling:}

End-labeled Primer $\quad 30 \mu \mathrm{l}$

\begin{tabular}{ll}
\hline Forward or Reverse primer $(6.6 \mu \mathrm{M})$ & $2 \mu \mathrm{l}$ \\
10X TMD & $3 \mu \mathrm{l}$ \\
10X BSA & $3 \mu \mathrm{l}$ \\
Water & $16 \mu \mathrm{l}$ \\
Gamma ${ }^{32} \mathrm{P}$ ATP & $5 \mu 1$ \\
Polynucleotide Kinase & $1 \mu \mathrm{l}$ \\
\hline
\end{tabular}

1. Make the 10X TMD solution with final concentrations of $500 \mathrm{mM}$ Tris $\mathrm{pH} 7.5,100 \mathrm{mM} \mathrm{MgCl}$, and $50 \mathrm{mM}$ DTT. Store at $20^{\circ} \mathrm{C}$.

2. Prepare the end-labeled primer by adding above reagents to a $1.5 \mathrm{ml}$ screw-cap tube for a $30 \mu \mathrm{l}$ total reaction volume. Incubate the reaction at $37^{\circ} \mathrm{C}$ for one hour.

3. Purify the sample with a G25 spin column from Roche ${ }^{\mathrm{TM}}$ according to the manufacturer's instructions. 


\begin{tabular}{ll}
\hline PCR Genotyping: & $\mathbf{1 m l}$ \\
10X Buffer 2 & \\
\hline $1 \mathrm{M} \mathrm{Tris} \mathrm{pH8.3}$ & $100 \mu \mathrm{l}$ \\
$1 \mathrm{M} \mathrm{KCl}$ & $500 \mu \mathrm{l}$ \\
$1 \mathrm{M} \mathrm{MgCl} 2$ & $20 \mu \mathrm{l}$ \\
$20 \mathrm{mg} / \mathrm{ml} \mathrm{BSA}$ & $50 \mu \mathrm{l}$ \\
Triton X & $5 \mu \mathrm{l}$ \\
Water & $325 \mu \mathrm{l}$ \\
\hline & \\
\hline Primer mix & $\mathbf{6 0 \mu l}$ \\
& \\
\hline Forward primer $(6.6 \mu \mathrm{M})$ & $10 \mu \mathrm{l}$ \\
Reverse primer $(6.6 \mu \mathrm{M})$ & $10 \mu \mathrm{l}$ \\
End-labeled primer $\left(0.44 \mu \mathrm{M}^{*}\right)$ & $40 \mu \mathrm{l}$ \\
${ }^{*}$ Concentration of end-labeled primer is only approximate. \\
\hline PCR reaction master mix & $\mathbf{1 0 \mu l}$ \\
\hline Template & $1 \mu \mathrm{l}$ \\
Water & $4.2 \mu \mathrm{l}$ \\
10Xbuffer2 & $1 \mu \mathrm{l}$ \\
2.5mM dNTPs & $1.6 \mu \mathrm{l}$ \\
Primer mix & $2 \mu \mathrm{l}$ \\
AmpliTaq DNA polymerase & $0.2 \mu \mathrm{l}$ \\
\hline
\end{tabular}

Prepare 10X Buffer 2 and store at $-20^{\circ} \mathrm{C}$. Primer mix should be made fresh before each genotyping experiment. Prepare master mix with an extra $10 \%$ of required volume to account for pipetting error. Aliquot $9 \mu 1$ of master mix per sample tube and amplify in MJ Research thermal cycler with the following conditions: $3 \mathrm{~min}$ at $94^{\circ} \mathrm{C}$ followed by 30 cycles of $94^{\circ} \mathrm{C}$ for $15 \mathrm{sec}, 55^{\circ} \mathrm{C}$ for $30 \mathrm{sec}$, then $72^{\circ} \mathrm{C}$ for $30 \mathrm{sec}$, followed by a final extension $72^{\circ}$ for 7 minutes. Be sure to include parental controls and combinations of parents to produce "artificial" UpDis and trisomies for scoring accuracy for reference on each gel.

\section{Electrophoresis}

\begin{tabular}{ll}
\hline Formamide Loading dye & $\mathbf{1 0 m l}$ \\
\hline Formamide & $9.5 \mathrm{ml}$ \\
Bromophenol Blue & $0.01 \mathrm{~g}$ \\
Xylene Cyanol FF & $0.01 \mathrm{~g}$ \\
\hline Store at $4^{\circ} \mathrm{C}$ & \\
\hline
\end{tabular}

1. Add $2 \mu$ of Formamide Loading dye to each sample and mix.

2. Prepare an $8 \%$ denaturing polyacrylamide gel using $25 \mathrm{~cm}$ glass sequencing plates with $0.3 \mathrm{~mm}$ spacers. Allow the gel to solidify for at least 1-2 hours. The gel may be left to set overnight as well.

3. Denature the PCR samples for $5 \mathrm{~min}$ at $95^{\circ} \mathrm{C}$ and place the tubes on ice. Load $3 \mu \mathrm{l}$ of each sample per lane and electrophorese the gel at $\sim 70$ Watts $(2000 \mathrm{~V}$ or $10 \mathrm{~V} / \mathrm{cm})$ for approximately 2 hours. Use Whatman paper to peel the gel from the plates and dry it on a vacuum gel dryer for approximately 1 hour until the moisture has been removed. Expose the dry gel to Kodak XOmat autoradiographic film at $-80^{\circ} \mathrm{C}$ overnight. Develop the film using a Kodak X-Omat film processor. Score the genotypes. 